\title{
Comparative Study of the Quadratic and Linear Shape Function Model in Cake Expression
}

\author{
Ndekwu O. B., Ademiluyi J. O. \\ Department of Civil Engineering, Faculty of Engineering, University of Nigeria, Nsukka
}

\begin{abstract}
The principle underlying expression basically involves the separation of a solid from the liquid in which it is suspended by passing the mixture through a porous medium with pore sizes too small to allow the passage of the solid particles at an applied pressure. The analytical method of the finite element method has been used to determine pressure, displacement and concentration distribution along sludge cake height in a filter press expression process. The ranges of displacement in a sludge cake height with increase time of expression increases with a decrease in sludge depth. The displacement increases continuously with increase time of expression. The finite element formulation basically involves the properties of the sludge in question as distinguished from other solution. The solution domain was idealized as a one-dimensional quadratic shape function for the purpose of this analysis and the displacement function formulation method were employed in solving the pressure distribution. The results of the finite element solution further showed that concentration increases with decreasing height of sludge cake interface during sludge dewatering. There is however an increasing tendency to uniformity of concentration with increment in the time of expression.
\end{abstract}

Keywords-Expression, Effective stress, Sludge cake.

\section{INTRODUCTION}

Solid-liquid separation consists of the separation of suspended solids from a liquid. This is often a multi-stage process that involves pre-treatment, solid concentration, solid separation, and post-treatment (Tien, 2012). One of the most popular methods of separation is filtration that is widely used in food and beverage, chemicals, petroleum, pharmaceutical, pulp and, and water and wastewater treatment industries. Filtration can be used either as the solid separation stage or as post-treatment stage (Rushton et al, 2000; Tien, 2012). For example, in wastewater treatment, filtration is employed in both primary and secondary treatment as well as bio-solids dewatering. Deeper understanding and fundamental study of the expression process would help to advance the filtration technology and to improve solid separation efficiency, increase filtrate flow rate, and lower the separation cost.

The sludge has high water content and compressibility attribute and as such it is expedient to dewater it to reduce its volume and prevent environmental health hazard. The dewatering of sludge using the filter press method has been in existence far back as in the 1920s. Since then, a number of equations have been presented by various contributors aimed at improving the performance of the sludge filtration/expression process, Carman, (1934), (1938); Grace, (1953); Coackley, (1956); Ademiluyi and others, (1983), (1987); Gale, (1975); Anazodo, (1974); Ademiluyi, (1986). However, their research was limited to experimental work which could not provide an insight into the interactive nature of sludge filterability. As previously sighted in literature (Anazodo, 1974; Ademiluyi, 1986) the inapplicability of Darcy's law of fluid flow to the dewatering process stems from the fact that Darcy's law is only applicable to rigid materials where porosity is constant. This uniformity in porosity implies a corresponding uniformity in displacement, pressure and concentration throughout the cake height. This is not true with compressible or deformable materials in for sludge cake. Porosity decreases from sludge to the cake height closest to the septum (Hemant, 1981; Bear et al, 1991; Lee et al, 2000; Svarosky, 2000; Challeppah et al, 2009; Challeppah et al, 2010). In the course of this porosity variation, displacement, pressure and concentration also vary. Even though the direction of porosity and all other parameters considered will be intuitively assessed, there is still the need to provide a theoretical base for such assessment. It is in the light of the foregoing that finite element method of analysis has been used in this research to evaluate the distribution displacement, pressure and concentration along the expressed cake height with a comparative review of the quadratic and linear shape function models. It is hoped that the study will find practical application in the evaluation of models necessary to describe cake expression phenomenon. 


\section{RESULTS AND DISCUSSION}

As presented in previous publications, the finite element model equation for pressure distribution using a displacement formulation matrix and concentration distribution has been developed for both the quadratic and linear shape function models. The quadratic shape function models prove to be more effective in describing the pattern of sludge cake displacement, pressure and concentration distribution at a constant pressure. As shown in figures (1, 2) below, the displacement plots seems to be exactly the same in both model cases. It is clear that as long as there are still void spaces and water pores in the sludge cake, displacement function will be on the increase as constant pressure pending on the effect of the hydraulic pressure.

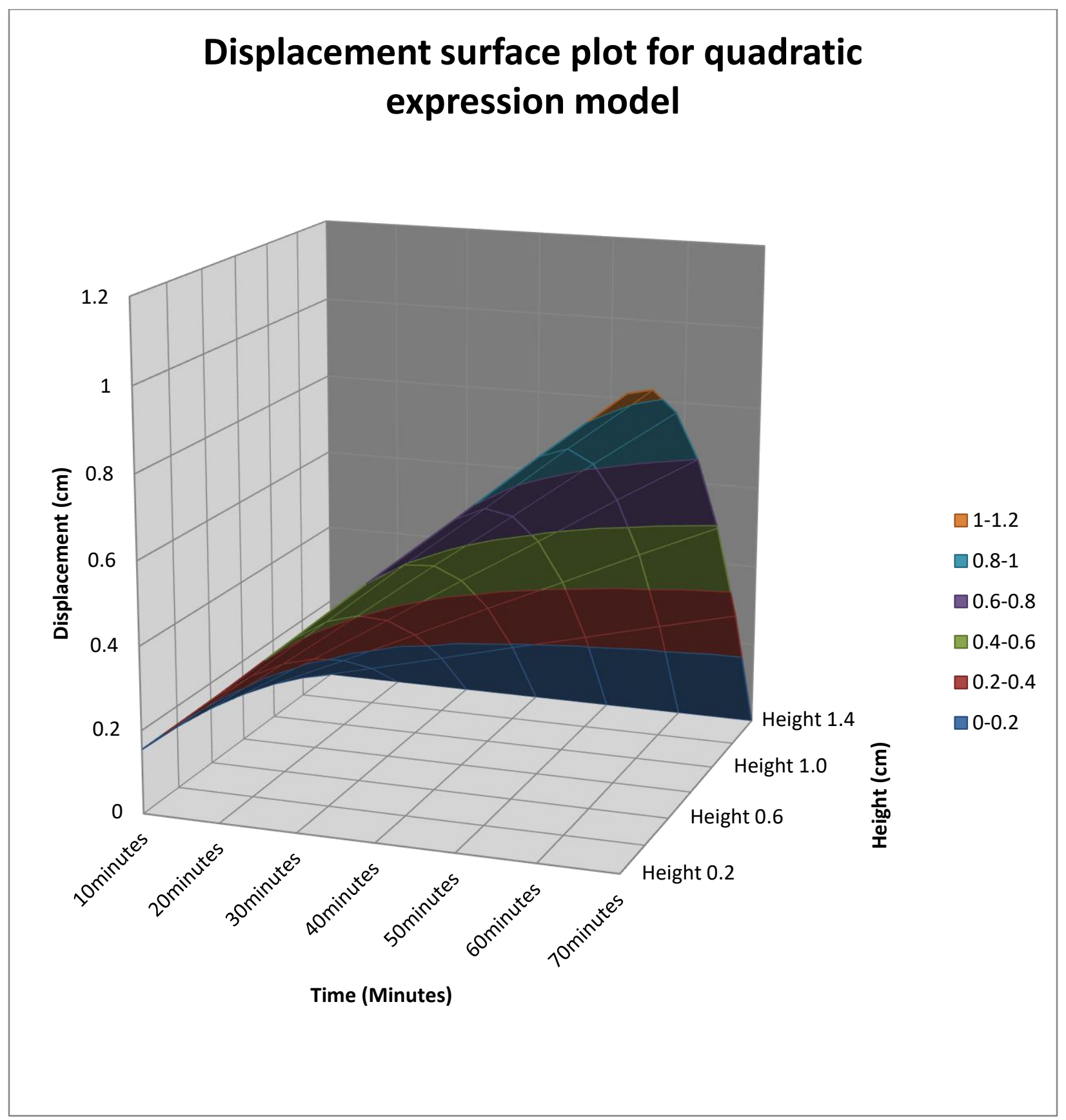

Fig.1: Surface plot for an expression displacement distribution of a quadratic function model. 


\section{Displacement surface plot for linear expression}

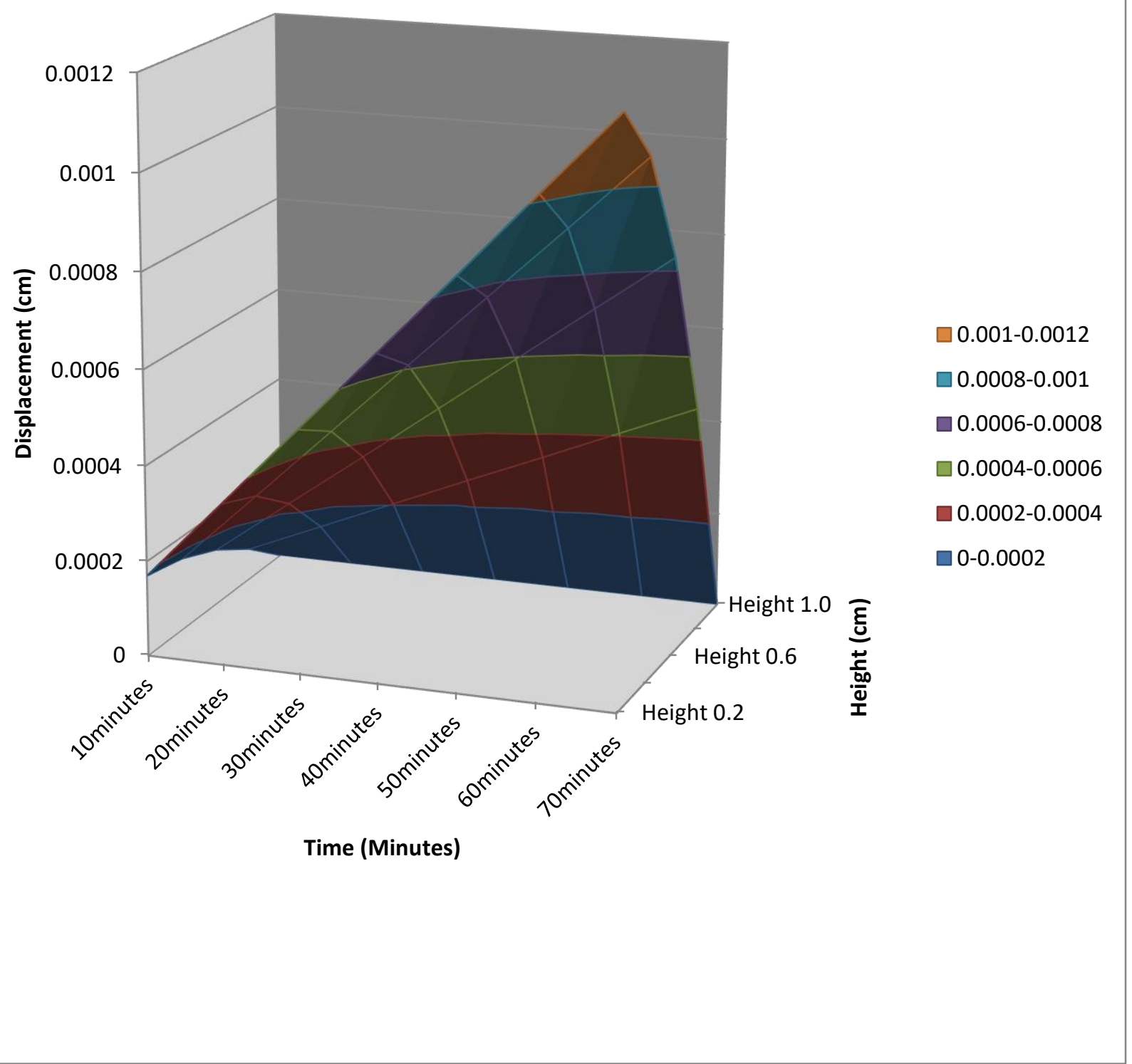

Fig.2: Surface plot for an expression displacement distribution of a linear function model.

The effective stress increases with decreasing height of sludge cake at different times of expression. The filter cake has an unstable structure, stabilized by some force related to flow velocity. In general an extremely loose packing exists and disturbances will easily cause changes in the structure. One of such disturbances is the unavoidable result of the process of flow. The drag force exerted by the liquid on the particles and on the filter material causes a cake pressure or filter medium pressure, which increase with the direction of flow. As shown in Figures 3 and 4 below, the curve of the quadratic function model depicts a typical expression case, conveying a picture of steady increases in pressure from the initial timing of 10 minutes to the final of 70 minutes. Its shows the relationship of the transition point between 
filtration and expression, hence confirming the initial plot of filtrate volume at varying time as a polynomial function.

But the linear function model has some discrepancies, though pressure buildup from the plot (Figure 4) is progressive, the uniformity does not really depict an expression phenomenon, hence branding the quadratic function model a better choice when designing an effective pressure distribution profile in cake expression.

\section{Effective stress surface plot for quadratic expression model}

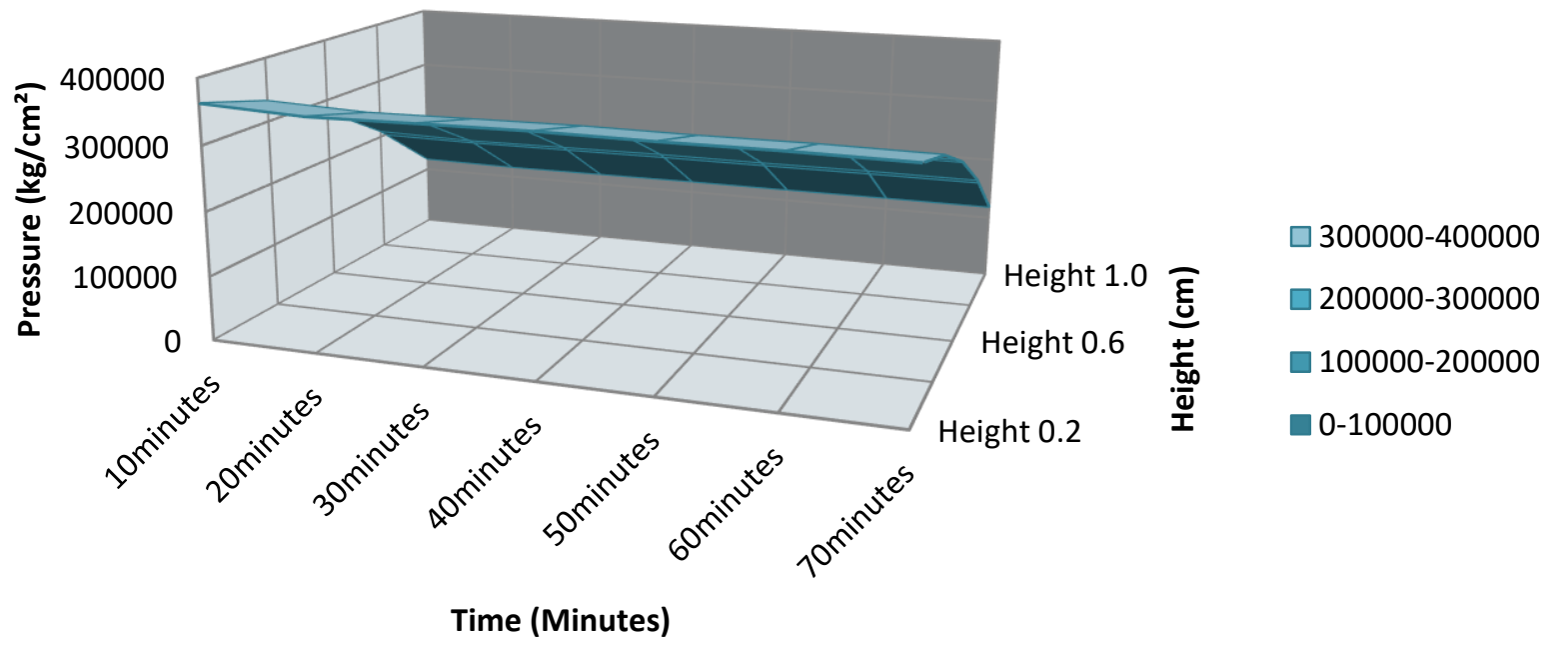

Fig.3: Surface plot for pressure distribution of a quadratic expression model

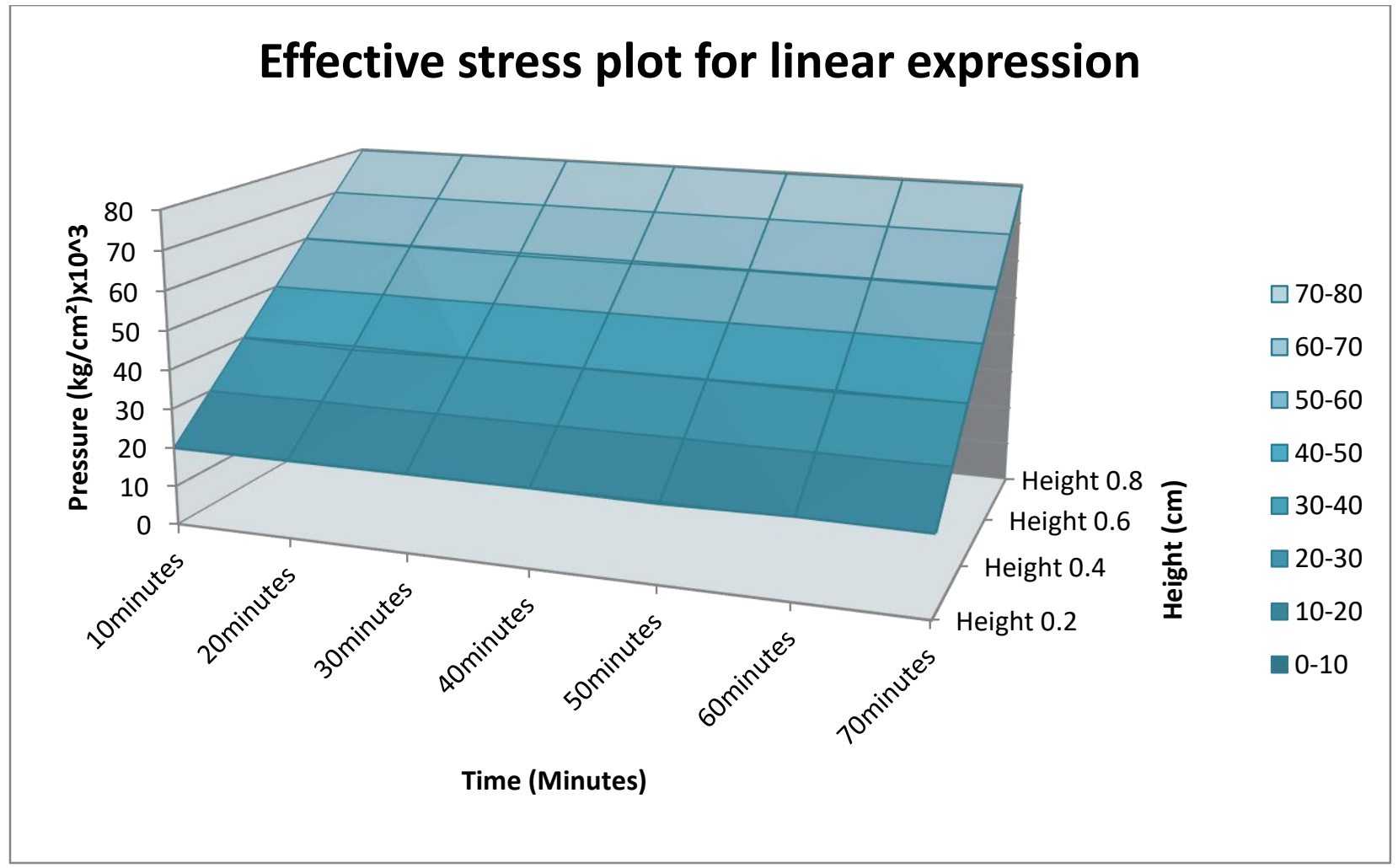

Fig.4: Surface plot for pressure distribution of a linear expression model 
It is evident, that from the plot of height of sludge versus pressure and displacement, there is a gradual trend to uniformity of pressure and displacement along the cake height as the time of expression increases. Hence the rate of displacement is directly proportional to the compressive pressure, which implies that the internal pressures are functions of both position and time. The effective pressure is not a physical quantity. The effective pressure is then simply the drag on all the particles in the distance from $\mathrm{x}$ to $\mathrm{L}$ divided by the cross-sectional area. This implies that the drop in hydraulic pressure from water pores across the sludge length resulting in an increase in displacement is exactly equal to the rise in solid compressive pressure.

However, the tendency to uniformity in concentration along the cake height is adjudged reasonable in the quadratic model plot as shown in figure (6) since it is expected that porosity decreases with time towards the filter septum. The linear model though tending to uniformity when considered on an average time of expression does not conform to the design expectation due to the outrageous values of concentration at certain times of expression. These occurrences could have theoretical explanations, but from the review of this study, it has been observed that the linear shape function model does not permit to a large extent the consideration of extra design location in the sludge cake, hence reducing the potency of the model from generating appropriate results that predicts the properties of the expressed cake.

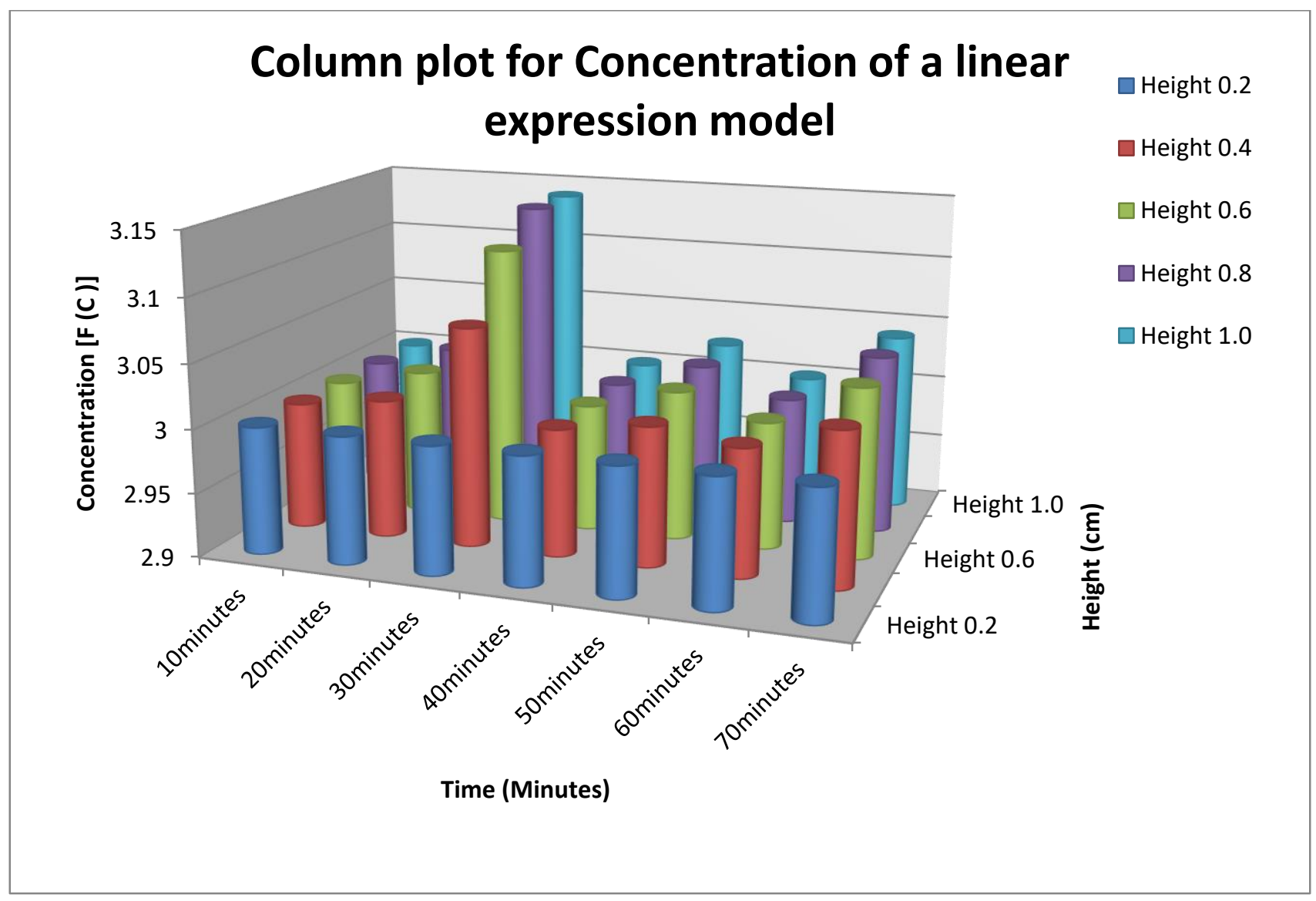

Fig.5: Column plot for concentration distribution of a linear expression model 


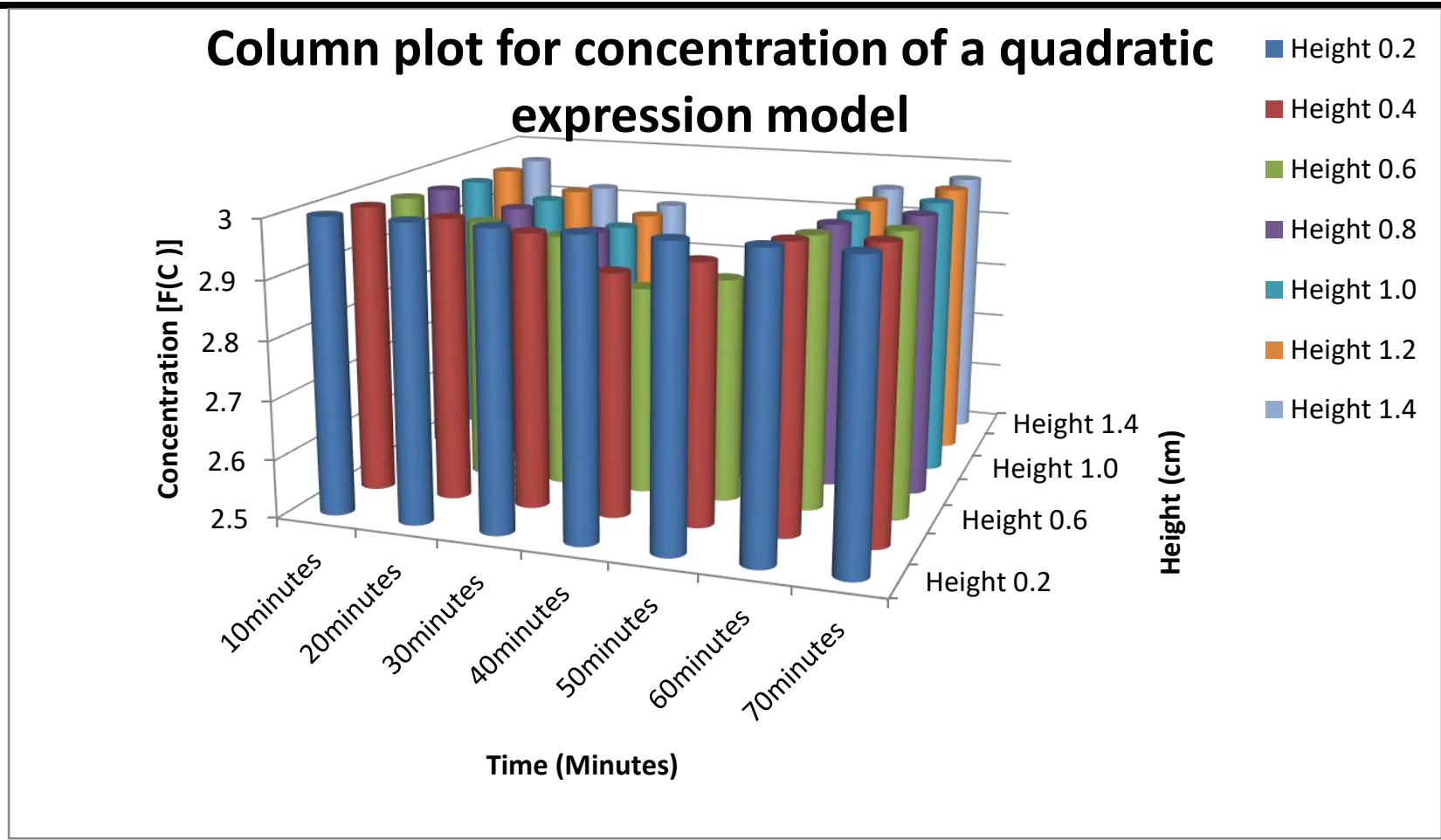

Fig.6: Column plot for concentration distribution of a quadratic expression model

\section{CONCLUSION}

From previous publications the results of the finite element solution have shown that;

1) Displacement along the sludge cake height increases with an increase in the effective pressure.

2) Effective stress increases with decreasing height along sludge cake at different expression time.

3) Concentration increases with decreasing height of sludge cake measured from sludge cake interface during sludge dewatering.

4) There is however an increase tendency to uniformity of concentration with increment of time.

All these conclusions were made possible from the results generated with the finite element linear and quadratic shape function models. But for industrial design purposes it is advised that more emphasis be based on the quadratic model due to its advantage of allowing more design points to be considered within the system environment being modeled.

\section{REFERENCES}

[1] Ademiluyi J. O. (1986). Development in the constant Vacuum cake filtration theory: Proceedings of the Engineering section of science association of Nigeria Vol. 6 \& 7.
[2] Ademiluyi, J. O. Egbuniwe, N. and Agunwamba, J. C. (1987). A dimensionless number as an index of sludge dewaterability, Journal of Engineering for Development, Vol. 1, p. 1-12.

[3] Ademiluyi, J.O. Anazodo, U.G. and Egbuniwe, N. (1983). Filterability and compressibility of sludge pt 11. Effluent and water treatment journal vol. 23, no 1 pp 25-30.

[4] Anazodo U.G. (1974). Dimensional equation for sludge filtration, effluent and water treatment journal vol, 14 n0 9 pp517-523.

[5] Bear, J. and Bachmat, Y. (1991), Introduction to Modeling of Transport Phenomena in Porous Media, Kluwer Academic Publishers.

[6] Carman, P.C. (1934). A Study of Mechanism of Filtration Part II, Journal of the Society of Chemical Industry, Transactions Communication. 53(6):15911651.

[7] Carman, P.C. (1938). Fundamental Principles of Industrial Filtration. Transactions - Institution of Chemical Engineers; 16: 168-188.

[8] Chellappah K. Tarleton E.S. and Wakeman R.J. (2009). Cake filtration and sedimentation of fibre/particle mixtures, Poster session presented at: 10th UK Particle Technology Forum, 2009 Jul 1-2; 
Birmingham, UK (won 2nd place in poster competition).

[9] Chellappah K. Tarleton E.S. and Wakeman R.J. (2010). The porosity, permeability and restructuring of heterogeneous filter cakes, Chemical Engineering and Technology (In Press).

[10] Coackley, P. and Jones, R.S. (1956). Vacuum sludge filtration, Sewage and industrial waste journal.

[11] Coprapciogh, Y. M. (1981). Formation of filter cakes, Filtration and separation, Vol. 18, No. 4, pp. 324-326.

[12] Gale, R.S. (1975). Comment on dimensional equation, Effluent and water treatment journal, p 422.

[13] Grace, H.P. (1953). Resistance and Compressibility of Filter Cakes, Part II: Under Conditions of Pressure Filtration, Chemical Engineering Progress, Vol. 49, No. 7, pp. 367-377.

[14] Hemant, R. M. (1981). Cakes filtration empirically incorporating parcile migration, Filtration and separation. Vol 18, No. 1,pp. 20-24.

[15] Lee, D. J. and Wang, C. H. (2000). Theories of cake filtration and consolidation and implications to sludge dewatering, Water Research, vol. 34(1), pp. 1-20

[16] Rushton, A., Ward, A. S. and Holdich, R. G. (2000). Solid-Liquid Filtration and Separation Technology. Weinheim: Wiley VCH.

[17] Svarovsky, L. ( 2000). Solid-Liquid Separation, 4th Ed., Butterworth-Heinemann, Oxford, UK,

[18] Tien, C. (2012). Principle of Filtration, Boston: Elsevier Science. 